\title{
Evaluación de resultados clínicos postoperatorios a corto y largo plazo de liberación percutánea con aguja vs técnica abierta de dedo en gatillo
}

\author{
Evaluation of postoperative results short and long term of percutaneous \\ liberation vs open technique for trigger finger
}

\author{
Carrasco-Ortiz O,* Pérez-Garmendia R, ${ }^{\ddagger}$ Márquez-Espriella C, ${ }^{\S}$ Arce-Salinas CA, „ \\ Dávila-Díaz R," Topete-González AO,** Garzón-Muvdi J,** Espino-Gaucín JI**
}

Hospital Central Sur de Alta de Especialidad de Petróleos Mexicanos.

RESUMEN. Introducción: El dedo en gatillo es muy frecuente en la población, con un riesgo de vida de padecer la enfermedad de $2.6 \%$ en la población general y con un aumento de 4 a $10 \%$ en diabéticos. Dado que no hay un estándar de oro de tratamiento quirúrgico y aún hay controversia en ello, es importante evaluar los resultados de las distintas técnicas quirúrgicas. El objetivo de este estudio es evaluar resultados postoperatorios de ambas técnicas quirúrgicas en pacientes con seguimiento de uno a 12 meses de postoperatorio. Material y métodos: Se trata de un estudio prospectivo, longitudinal, descriptivo y observacional realizado en un período de Enero de 2015 a Diciembre de 2017. Se incluyeron pacientes postoperados de liberación con técnica abierta (grupo 1) y percutánea con aguja (grupo 2). Se llevó a cabo la revisión de expedientes de todos los pacientes y se les hizo una encuesta mediante vía telefónica. Los resultados de la comparación de ambas técnicas se analizaron mediante $\chi^{2}$ para resultados paramétricos y mediante la prueba de Fisher para los no paramétricos. Resultados: Se encontró que los pacientes del grupo 2 manifestaron mayor satisfacción, de los cuales $21.8 \%(n=12)$ estuvieron totalmente satisfechos con el procedimiento percutáneo, a
ABSTRACT. Indroduction: Trigger finger is very common in the population, with a life-threatening risk of developing the disease of $2.6 \%$ in the general population and increasing to 4 to $10 \%$ in diabetics. Since there is no standard gold of surgical treatment and there is still controversy in this, it is important to evaluate the results of the different surgical techniques. The objective of this study is to evaluate postoperative results of both surgical techniques in patients with follow-up of 1 to 12 months postoperative. Material and methods: It is a prospective, longitudinal, descriptive and observational study carried out in a period from January 2015 to December 2017. Postoperative open (group 1) and percutaneous needle (group 2) patients were included. All patients were reviewed and surveyed by telephone. The comparison results of both techniques were analyzed using $\chi^{2}$ for parametric results and by the Fisher test for nonparametric results. Results: It was found that patients in group 2 expressed greater satisfaction, where $21.8 \%(n=12)$ were fully satisfied with the percutaneous procedure, unlike those in group 1 where total satisfaction was only manifested at 3.8\% ( $\mathrm{n}=$ 2). Conclusions: In this study we can conclude that both

\section{Nivel de evidencia: II}

* Médico residente de tercer año de Cirugía Plástica y Reconstructiva.

‡ Investigadora Escuela de Medicina Universidad Panamericana.

$\S$ Jefe de Servicio de Cirugía Plástica y Reconstructiva.

" Jefe de Servicio de Medicina Interna y Asesor Estadístico.

" Médico adscrito al Servicio de Cirugía Plástica y Reconstructiva.

** Médico residente de Cirugía Plástica y Reconstructiva.

Dirección para correspondencia:

Olin Carrasco-Ortiz

Periférico Sur Núm. 4091,

Col. Fuentes del Pedregal,

Alcaldía Tlalpan, CP. 14140, Ciudad de México, México.

E-mail: mxcu66@gmail.com 
diferencia de los del grupo 1 quienes manifestaron satisfacción total sólo en 3.8\% $(n=2)$. Conclusiones: En este estudio podemos concluir que ambas técnicas son efectivas para el tratamiento de dedo en gatillo, siendo la liberación percutánea con aguja la que ofrece mayor grado de satisfacción en los pacientes.

Palabras clave: Dedo en gatillo, tratamiento, percutáneo, abierto, resultados, satisfacción. techniques are effective for the treatment of trigger finger, with percutaneous needle release offering the highest degree of satisfaction in patients.

Keywords: Trigger finger, treatment, percutaneous, open, results, satisfaction.

\section{Introducción}

La enfermedad de dedo en gatillo es muy frecuente en la población, con un riesgo de vida de padecer la enfermedad de $2.6 \%$ en la población general y con un aumento de 4 a $10 \%$ en población diabética. ${ }^{1}$

El atrapamiento de tendones flexores, también conocido como dedo en gatillo o tenosinovitis estenosante, es una condición que causa engatillamiento, chasquido o atrapamiento en flexión del dedo involucrado.

Las vainas tendinosas flexoras en el dedo consisten en un cilindro de tejido conectivo de doble pared, localizadas en cuatro poleas anulares; (A1, A4) y tres poleas cruzadas (C1-C3). El fenómeno de engatillamiento es causado por incompatibilidad entre el tendón y su vaina, probablemente debido a hipertrofia de la polea A1.

Desde el punto de vista histológico la polea A1 muestra hallazgos consistentes con metaplasia fibrocartilaginosa, mayor número de condrocitos, de glucosaminoglicanos, cambios proliferativos y tejido fibroso. ${ }^{2}$

El dedo en gatillo se clasifica en cuatro grados de acuerdo a la clasificación de Green.

Afecta a un buen porcentaje de nuestra población, trabajadores o no, ambos géneros y a distintos grupos de edad. Por otra parte, esta patología repercute en la calidad de vida y en las actividades tanto personales como laborales en cada paciente. Es una de las patologías de mano más frecuentes y generalmente requiere de tratamiento quirúrgico.

Tan sólo en nuestro hospital en los últimos cinco años se realizó un total de 88 cirugías de liberación de dedo en gatillo, nueve reintervenciones y 79 cirugías de primera vez, de las cuales 29 pacientes son trabajadores activos y 23 son jubilados y los 36 pacientes restantes son familiares.

Hay diversos tratamientos para el dedo en gatillo, tanto manejo conservador como quirúrgico. Dentro del manejo conservador hay diversas opciones: modificaciones en las actividades, uso de férulas, fisioterapia, uso de AINES, entre otros; sin embargo, la principal modalidad de tratamiento es el uso de corticosteroides. Diversos estudios han mostrado éxito con el uso de corticosteroides hasta en 6184\% de pacientes que se presentan con síntomas de dedo en gatillo de reciente diagnóstico posterior a una o tres inyecciones. $^{3,4,5,6,7,8,9,10,11}$ Sin embargo, la recurrencia de dedo en gatillo con el uso de esteroides es de $27 \%$ en un año de seguimiento, lo que sugiere que aunque el uso de esteroides es útil, no provee remisión de la sintomatología a largo plazo. ${ }^{8}$ Las tasas de fallas se han relacionado con pacientes jóvenes, diabetes mellitus, el incremento en la duración de los síntomas y la afección de múltiples dedos. ${ }^{12}$

Dentro de las técnicas quirúrgicas hay varias opciones: técnica abierta, percutánea con aguja y con técnica convencional. Todas tienen sus ventajas y desventajas, teniendo un amplio rango de porcentajes de efectividad en todos los tipos de tratamiento dependiendo de las series y trabajos publicados. Dado que no hay un estándar de oro de tratamiento quirúrgico y aún hay controversia en ello, es importante evaluar los resultados de las distintas técnicas quirúrgicas.

El objetivo de este estudio es evaluar los resultados postoperatorios de dos técnicas quirúrgicas en pacientes con seguimiento de uno a 12 meses del postoperatorio.

\section{Material y métodos}

Se trata de un estudio prospectivo, longitudinal, descriptivo y observacional realizado en un período de Enero de 2015 a Diciembre de 2017.

Se llevó a cabo la revisión de expedientes de todos los pacientes postoperados de liberación de dedo en gatillo con técnica percutánea con aguja o mediante técnica abierta.

Posteriormente se les hizo una encuesta mediante vía telefónica a todos los pacientes que cumplieran los criterios de inclusión y que dieran su consentimiento para contestarla. Las encuestas se realizaron en el transcurso de dicho período.

Se evaluaron datos clínicos postoperatorios; sensibilidad, dolor, gatilleo, nódulo o induración a nivel de polea A1 y el grado de satisfacción.

Se dividieron en dos grupos, el grupo 1 con pacientes postoperados de liberación con técnica abierta, y el grupo 2 con pacientes postoperados de liberación percutánea con aguja. Todos los pacientes tuvieron un seguimiento postoperatorio entre uno y 12 meses.

Se usó el programa SPSS versión 24 para el análisis estadístico. Los resultados de la comparación de ambas técnicas se analizaron mediante $\chi^{2}$ para resultados paramétricos y mediante la prueba de Fisher para los no paramétricos.

Criterios de inclusión: pacientes postoperados de liberación de dedo en gatillo con ambas técnicas, abierta y per- 
cutánea con aguja, que no hayan recibido tratamiento conservador previamente, con uno a 12 meses de seguimiento postoperatorio que puedan ser localizados vía telefónica, que quieran responder la encuesta y den su consentimiento y que puedan entender y contestar adecuadamente la encuesta.

Criterios de exclusión: pacientes postoperados de liberación percutánea convencional de dedo en gatillo, con diagnóstico de dedo en gatillo tratados mediante tratamiento conservador, con esteroides, férulas o sólo con rehabilitación física, con otra patología en mano afectada y con cirugía previa en la misma.

Criterios de eliminación: pacientes que no contesten la encuesta y pacientes finados.

Técnica quirúrgica abierta: consiste en una incisión de 5-8 mm aproximadamente de largo, a nivel de la polea A1 del dedo afectado, disección de tejido celular subcutáneo, incisión a lo largo de toda la polea A1 verificando la liberación del tendón flexor, así como la hemostasia, posteriormente se comprueba de forma transoperatoria la liberación exitosa del tendón flexor. Al final se cierra piel con sutura monofilamente no absorbible 4-0.

Técnica percutánea con aguja: consiste en la introducción perpendicular a la piel de una aguja de 20G a 2-3 mm distal al borde proximal y en el centro de la polea A1 teniendo cuidado de no desviarse de la línea media de la polea con el fin de no lesionar el paquete neurovascular. Se comprueba la introducción de la aguja en el tendón movilizando la articulación interfalángica distal al mismo tiempo que observamos el desplazamiento de la aguja. Se retira lentamente hasta no ver dicho movimiento, en este momento la aguja se encuentra dentro de la polea A1. Ésta se corta moviendo la aguja de proximal a distal y se corrobora la liberación completa de la polea moviendo el dedo en flexión y extensión corroborando la desaparición del engatillamiento. ${ }^{13}$

\section{Resultados}

Se operó un total de 107 pacientes, 52 con técnica abierta de liberación de dedo en gatillo (grupo 1) y 55 con técnica percutánea con aguja (grupo 2) en el período del 01 de Enero de 2015 al 31 de Diciembre de 2017. Se revisó el expediente electrónico de todos los pacientes. Se observó que en ambos grupos hubo un predominio de mujeres (grupo $1=$ $69.2 \%$ vs grupo $2=69.1 \%$ ) con respecto a hombres (grupo $1=30.8 \%$ vs grupo $2=39.9 \%)$. No se encontraron diferencias con respecto a la edad. Los pacientes tuvieron un seguimiento en promedio de 3.19 meses $( \pm 3.16)$ (Tabla 1$)$.

Observamos que el dedo más comúnmente afectado, en ambos grupos, fue el tercero (grupo $1=36.5 \%$ vs grupo 2 $=32.7 \%$ ). La prevalencia de diabetes mellitus tipo 2 en el grupo 1 fue de $23.1 \%$, mientras que en el grupo 2 fue de $40 \%$ (Tabla 1).

En cuanto a la sintomatología, hubo recurrencia en $26.9 \%(n=14)$ en el grupo 1 y $25.5 \%(n=14)$ en el grupo 2 . Al evaluar la sensibilidad no se encontró diferencia significativa entre los grupos. Con respecto al dolor $21.2 \%(n=$ 11) del grupo 1 refirió dolor leve, mientras que en el grupo 2 el dolor se refirió en $16.4 \%(\mathrm{n}=9) .3 .8 \%(\mathrm{n}=2)$ de los pacientes del grupo 1 refirieron dolor moderado, mientras

\begin{tabular}{|c|c|c|c|c|c|c|}
\hline & & \multicolumn{4}{|c|}{ Cirugía realizada } & \multirow[b]{3}{*}{$\mathrm{p}$} \\
\hline & & \multicolumn{2}{|c|}{ Abierta } & \multicolumn{2}{|c|}{ Percutánea } & \\
\hline & & $\mathrm{n}$ & $\%$ & $\mathrm{n}$ & $\%$ & \\
\hline \multirow[t]{7}{*}{ Género } & Hombre & 16 & 30.8 & 17 & 30.9 & \\
\hline & Mujer & 36 & 69.2 & 38 & 69.1 & \\
\hline & 1 & 20 & 38.5 & 16 & 29.1 & \\
\hline & 2 & 3 & 5.8 & 6 & 10.9 & \\
\hline & 3 & 19 & 36.5 & 18 & 32.7 & \\
\hline & 4 & 9 & 17.3 & 15 & 27.3 & \\
\hline & 5 & 1 & 1.9 & 0 & 0.0 & \\
\hline \multirow[t]{2}{*}{ Sintomatología } & Asintomático & 38 & 73.1 & 41 & 74.5 & 0.58 \\
\hline & Recurrente & 14 & 26.9 & 14 & 25.5 & \\
\hline \multirow[t]{11}{*}{ Seguimiento (meses) } & 0 & 0 & 0.0 & 2 & 3.6 & \\
\hline & 1 & 28 & 53.8 & 17 & 30.9 & \\
\hline & 2 & 8 & 15.4 & 7 & 12.7 & \\
\hline & 3 & 2 & 3.8 & 8 & 14.5 & \\
\hline & 4 & 4 & 7.7 & 6 & 10.9 & \\
\hline & 5 & 0 & 0.0 & 1 & 1.8 & \\
\hline & 6 & 2 & 3.8 & 8 & 14.5 & \\
\hline & 7 & 2 & 3.8 & 0 & 0.0 & \\
\hline & 8 & 1 & 1.9 & 0 & 0.0 & \\
\hline & 10 & 3 & 5.8 & 3 & 5.5 & \\
\hline & 12 & 2 & 3.8 & 3 & 5.5 & \\
\hline \multirow[t]{2}{*}{ Diabetes mellitus } & Ausente & 40 & 76.9 & 33 & 60.0 & 0.06 \\
\hline & Presente & 12 & 23.1 & 22 & 40.0 & \\
\hline
\end{tabular}




\begin{tabular}{|c|c|c|c|c|c|c|}
\hline & & \multicolumn{4}{|c|}{ Cirugía realizada } & \multirow[b]{3}{*}{$\mathrm{p}$} \\
\hline & & \multicolumn{2}{|c|}{ Abierta } & \multicolumn{2}{|c|}{ Percutánea } & \\
\hline & & $\mathrm{n}$ & $\%$ & $\mathrm{n}$ & $\%$ & \\
\hline \multirow[t]{3}{*}{ Sensibilidad } & Frecuente & 1 & 1.9 & 3 & 5.5 & \\
\hline & Nunca & 47 & 90.4 & 45 & 81.8 & 0.400 \\
\hline & Ocasional & 4 & 7.7 & 7 & 12.7 & \\
\hline \multirow[t]{3}{*}{ Dolor } & Ausente & 39 & 75.0 & 44 & 80.0 & \\
\hline & Leve & 11 & 21.2 & 9 & 106.4 & 0.812 \\
\hline & Moderado & 2 & 3.8 & 2 & 3.6 & \\
\hline \multirow[t]{3}{*}{ Gatilleo } & Grado 1 & 7 & 13.5 & 9 & 16.4 & \\
\hline & Grado 2 & 6 & 11.5 & 5 & 9.1 & 0.858 \\
\hline & Ausente & 39 & 75.0 & 41 & 74.5 & \\
\hline \multirow[t]{2}{*}{ Nódulo } & Ausente & 45 & 86.5 & 47 & 85.5 & 0.547 \\
\hline & Presente & 7 & 13.5 & 8 & 14.5 & \\
\hline \multirow[t]{4}{*}{ Satisfacción } & Insatisfecho & 0 & 0.0 & 1 & 1.8 & \\
\hline & Muy satisfecho & 42 & 80.8 & 34 & 61.8 & 0.031 \\
\hline & Poco satisfecho & 8 & 15.4 & 8 & 14.5 & \\
\hline & Totalmente satisfecho & 2 & 3.8 & 12 & 21.8 & \\
\hline
\end{tabular}

que en el grupo 2, 3.6\% $(n=2)$ lo refirieron. Las diferencias no fueron estadísticamente significativas.

$\mathrm{Al}$ evaluar la presencia de gatilleo se observó que $13.5 \%$ $(\mathrm{n}=7)$ de los pacientes del grupo 1 presentaron un gatilleo grado 1 y $11.5 \%(n=6)$ gatilleo grado 2 , mientras los pacientes del grupo 2 los presentaron en $16.4 \%(n=9)$ y $9.1 \%$ $(n=5)$, respectivamente. Las diferencias no alcanzaron significancia estadística.

En $13.5 \%(n=7)$ de los pacientes del grupo 1 presentaron un nódulo a nivel polea A1 del dedo afectado comparado con $14.5 \%(\mathrm{n}=8)$ del grupo 2 .

Al comparar los grupos se observó que los pacientes del grupo 2 manifestaron mayor satisfacción, de los cuales $21.8 \%(n=12)$ estuvieron totalmente satisfechos con el procedimiento percutáneo, a diferencia de los pacientes del grupo 1 en quienes la satisfacción total sólo se manifestó en $3.8 \%(\mathrm{n}=2)($ Tabla 2$)$.

\section{Discusión}

En una revisión sistemática, actualmente en prensa, en relación a la efectividad en los distintos tipos de tratamiento quirúrgico en dedo en gatillo, Bionka y colaboradores concluyen que hay poca evidencia y que ésta es controversial en relación a una u otra técnica a corto plazo. ${ }^{14}$

Gilberts encuentra diferencias significativas a favor de la técnica percutánea en cuanto a menos duración del dolor postoperatorio $(\mathrm{p}=0.39)$ y recuperación de la función motora a 12 semanas de seguimiento $(p=0.002)$ sin mostrar diferencias significativas en tasa de éxito. ${ }^{15}$

Dierks muestra diferencia significativa en rango de movimiento activo de la articulación interfalángica proximal en favor de la técnica percutánea a una semana postoperatoria $(\mathrm{p}<0.05)$, pero no detecta diferencia en fuerza de agarre y dolor a 12 semanas. ${ }^{16}$ En ambos casos mencionados pre- viamente son resultados a corto plazo, Gilberts a 12 semanas y Dierks a una semana, a diferencia de nuestro estudio que muestra resultados a largo plazo.

Bamroongshawgasame tampoco encontró diferencias en movimientos de flexión y extensión postoperatorios luego de tres a ocho semanas de seguimiento. ${ }^{17}$

Topper comparó división parcial por tercios de la polea A1, en proximal, medio y distal, sin observar diferencias significativas. $^{18}$

Éste es uno de los pocos estudios que evalúan resultados a largo plazo comparando dos técnicas quirúrgicas. Por otra parte, son pocos los estudios que reportan resultados con técnica percutánea con aguja. Nuestro estudio arroja resultados similares a los descritos en la literatura.

Una limitante del estudio es que no analizamos el descontrol metabólico de los pacientes diabéticos ni la influencia de las comorbilidades de cada paciente; por otra parte, no es un estudio clínico aleatorizado. En este trabajo podemos concluir que ambas técnicas son efectivas para el tratamiento de dedo en gatillo, siendo la liberación percutánea con aguja la técnica que ofrece mayor grado de satisfacción en los pacientes.

Bibliografía

1. Robert D. Wojahn, MD, Nicholas C. Foeger, MD. Long-term outcomes following a single corticosteroid injection for trigger finger. J Bone Joint Surg Am. 2014; 96(229: 1849-54.

2. Moore JS. Flexor tendon entrapment of the digits (trigger finger and trigger thumb). J Occup Environ Med. 2000; 42(5): 526-45.

3. Patel MR, Bassini L. Trigger fingers and thumb: when to splint, inject, or operate. J Hand Surg Am. 1992; 17(1): 110-3.

4. Kolind-Sorensen V. Treatment of trigger fingers. Acta Orthop Scand. 1970; 41(4): 428-32.

5. Newport ML, Lane LB, Stuchin SA. Treatment of trigger finger by steroid injection. J Hand Surg Am. 1990; 15(5): 748-50.

6. Rhoades CE, Gelberman RH, Manjarris JF. Stenosing tenosynovitis of the fingers and thumb. Results of a prospective trial of steroid injection and splinting. Clin Orthop Relat Res. 1984; (190): 236-8. 
7. Clark DD, Ricker JH, MacCollum MS. The efficacy of local steroid injection in the treatment of stenosing tenovaginitis. Plast Reconstr Surg. 1973; 51(2): 179-80.

8. Anderson B, Kaye S. Treatment of flexor tenosynovitis of the hand ('trigger finger') with corticosteroids. A prospective study of the response to local injection. Arch Intern Med. 1991; 151(1): 153-6.

9. Faunø P, Andersen HJ, Simonsen O. A long-term follow-up of the effect of repeated corticosteroid injections for stenosing tenovaginitis. J Hand Surg Br. 1989; 14(2): 242-3.

10. Marks MR, Gunther SF. Efficacy of cortisone injection in treatment of trigger fingers and thumbs. J Hand Surg Am. 1989; 14(4): 722-7.

11. Freiberg A, Mulholland RS, Levine R. Nonoperative treatment of trigger fingers and thumbs. J Hand Surg Am. 1989; 14(3): 553-8.

12. Wojahn RD, Foeger NC. Long-term outcomes following a single corticosteroid injection for trigger finger. J Bone Joint Surg Am. 2014; 96: 1849-54.

13. Mishra SR, Gaur AK, Choudhary MM, Ramesh J. Percutaneous A1 pulley release by the tip of a 20-g hypodermic needle before open surgical procedure in trigger finger management. Tech Hand Up Extrem Surg. 2013; 17(2): 112-5.

14. Huisstede BM, Gladdines S, Randsdorp MS, Koes BW. Effectiveness of conservative, surgical, and postsurgical interventions for trigger finger, Dupuytren disease, and de quervain disease: a systematic review. Arch Phys Med Rehabil. 2018; 99(8): 1635-1649.e21.
15. Gilberts EC, Beekman WH, Stevens HJ, Wereldsma JC. Prospective randomized trial of open versus percutaneous surgery for trigger digits. J Hand Surg Am. 2001; 26: 497-500.

16. Dierks U, Hoffmann R, Meek MF. Open versus percutaneous release of the A1-pulley for stenosing tendovaginitis: a prospective randomized trial. Tech Hand Up Extrem Surg. 2008; 12: 183-7.

17. Bamroongshawgasame T. A comparison of open and percutaneous pulley release in trigger digits. J Med Assoc Thai. 2010; 93(2): 199204.

18. Topper SM, Jones DE, Klajnbart JO, Friedel SP. Trigger finger: the effect of partial release of the first annular pulley on triggering. Am $J$ Orthop (Belle Mead NJ). 1997; 26: 675-7.

Comité de ética e investigación: Aprobado por los comités de ética e investigación de la institución donde se llevó a cabo el estudio.

Cesión de derechos y acuerdos con el autor a la revista.

Conflicto de intereses: No existe ningún conflicto de intereses en el momento que se envía el manuscrito o el artículo a la Revista Acta Ortopédica Mexicana. 